\title{
Rapid determination of anti-tuberculosis drug resistance from whole-genome sequences
}

\author{
Francesc Coll ${ }^{1}$, Ruth McNerney ${ }^{1}$, Mark D Preston ${ }^{1}$, José Afonso Guerra-Assunção ${ }^{1}$, Andrew Warry², \\ Grant Hill-Cawthorne ${ }^{3,4}$, Kim Mallard', Mridul Nair ${ }^{3}$, Anabela Miranda ${ }^{5}$, Adriana Alves ${ }^{5}$, João Perdigão ${ }^{6}$, Miguel Viveiros ${ }^{7}$, \\ Isabel Portugal ${ }^{6}$, Zahra Hasan ${ }^{8}$, Rumina Hasan ${ }^{8}$, Judith R Glynn ${ }^{1,9}$, Nigel Martin ${ }^{10}$, Arnab Pain $^{3^{*}}$ and Taane G Clark ${ }^{1 *}$
}

\begin{abstract}
Mycobacterium tuberculosis drug resistance (DR) challenges effective tuberculosis disease control. Current molecular tests examine limited numbers of mutations, and although whole genome sequencing approaches could fully characterise DR, data complexity has restricted their clinical application. A library (1,325 mutations) predictive of DR for 15 anti-tuberculosis drugs was compiled and validated for 11 of them using genomic-phenotypic data from 792 strains. A rapid online TB-Profiler' tool was developed to report DR and strain-type profiles directly from raw sequences. Using our DR mutation library, in silico diagnostic accuracy was superior to some commercial diagnostics and alternative databases. The library will facilitate sequence-based drug-susceptibility testing.
\end{abstract}

\section{Background}

Resistance has been reported to all drugs used to treat tuberculosis (TB) [1]. Increased resistance is associated with decreased patient survival and is a substantial threat to disease control. The World Health Organization (WHO) classifies tuberculosis resistant to isoniazid and rifampicin as multi drug-resistant (MDR-TB), when a switch to second line treatment is advised. Resistance to additional drugs further compromises treatment success [2]. MDR-TB strains that have developed resistance to the fluoroquinolones and aminoglycosides are classed as extensively drug resistant (XDR-TB). The term totally drug resistant (TDR-TB) has been used to describe strains found resistant to all available drugs, but there is not yet an agreed definition of TDR-TB [1]. Treatment of drug resistant disease is prolonged and expensive, and outcomes are poor $[2,3]$. Treatment involves drugs of heightened toxicity and adverse reactions are common and may be severe and irreversible $[4,5]$. Poor tolerance leads to reduced compliance, which in turn reduces cure rates and can result in amplification of resistance [6].

\footnotetext{
* Correspondence: arnab.pain@kaust.edu.sa; taane.clark@lshtm.ac.uk ${ }^{3}$ Biological and Environmental Sciences and Engineering Division, King Abdullah University of Science and Technology (KAUST), Thuwal, Kingdom of Saudi Arabia

${ }^{1}$ Faculty of Infectious and Tropical Diseases, London School of Hygiene \& Tropical Medicine, Keppel Street, London WC1E 7HT, UK

Full list of author information is available at the end of the article
}

Early detection is crucial for access to effective treatment and prevention of onward transmission. Knowledge of the full drug susceptibility profile would enable tailored treatment to improve efficacy and reduce exposure to ineffective toxic drugs. Current testing for resistance to most anti-TB drugs involves isolation and culture of the bacteria followed by exposure to the drug, a process that takes weeks or months and requires high levels of microbiological safety. The primary cause of resistance in $M$. tuberculosis is the accumulation of point mutations and insertions and deletions (indels) in genes coding for drug-targets or -converting enzymes [7]. Rapid molecular assays that test directly from sputum are available for some key drugs. In 2013 the Xpert MTB/RIF (Cepheid, Inc., Sunnyvale, CA, USA), was granted US FDA approval for detecting resistance to rifampicin, conditional on confirmatory testing [8]. This easy to use semi-automated PCR-based test has also been endorsed by WHO, as have Line Probe Assays (LPA) for resistance to rifampicin and isoniazid, where, following amplification of bacterial DNA, samples are interrogated with a panel of oligonucleotide probes [9]. LPA to detect resistance to other drugs, including fluoroquinolones and aminoglycosides, have also been developed [10], but have yet to be endorsed by WHO. Though undoubtedly useful, both technologies are limited in the number of loci they examine and they lack 
capacity to differentiate silent mutations from those that effect drug efficacy, leading to false positive results [11-13]. Whole genome sequencing has the potential to overcome such problems and extend rapid testing to the full range of anti-TB drugs and has been applied in a clinical setting. Bench top analysers have been developed capable of sequencing a bacterial genome in a few hours and costs have been greatly reduced with the introduction of high throughput technology. Sequencing already assists patient management for a number of conditions such as HIV for which Sanger sequencing is performed to determine viral tropism and drug susceptibility [14]. Recent reports of sequencing $M$. tuberculosis from sputum from suspected XDR-TB patients suggests it has a role in the management of TB [15-17]. However, data analysis remains a bottleneck, requiring specialist expertise not readily available in clinical laboratories. To address this issue and progress sequencing towards real time management of patients we have compiled an exhaustive library of 1,325 drug resistance markers and developed an online tool that rapidly analyses raw sequence data and predicts resistance. We present accuracy data comparing in silico whole genome analysis for resistance to 11 anti-TB drugs, to conventional drug susceptibility testing (DST). To further assess potential benefits of the whole genome approach we compared our curated mutation database to two others (TBDreaMDB and MUBII-TB-DB), as well as those used in three commercial molecular tests, the Xpert MTB/RIF (Cepheid, Inc., Sunnyvale, CA, USA), and the MTBDRplus and MTBDRsl (Hain Life Science, Germany). In particular, in silico versions of the three commercial molecular tests were implemented.

\section{Methods}

\section{Mutation library}

Following review of available data, a library of mutations predictive of drug resistance was compiled. First, mutations from two publically available web-based tools $T B D r e a M D B$ [18] and $M U B I I-T B-D B$ [19] were extracted. Second, phylogenetic SNPs at drug resistance loci were removed (see Additional file 1: Table S2 for the full list), as they have been historically misclassified as drug resistance markers [20,21]. And third, recent literature was consulted to include mutations and loci not described in TBDreaMDB and MUBII-TB-DB. (See Additional file 1: Table S1 for a list of source materials). Drugs included were amikacin (AMK), capreomycin (CAP), ethambutol (EMB), ethionamide (ETH), isoniazid (INH), kanamycin (KAN), moxifloxacin (MOX), ofloxacin (OFX), pyrazinamide (PZA), rifampicin (RMP), streptomycin (STR), para-aminosalicylic acid (PAS), linezolid (LZD), clofazimine (CFZ) and bedaquiline (BDQ). As presented in Table 1, the library comprised 1,325 polymorphisms
Table 1 Summary of mutations included in the curated whole genome drug resistance library

\begin{tabular}{|c|c|c|c|c|}
\hline Drug & Loci & No. variable sites & SNPs & Indels \\
\hline \multirow[t]{7}{*}{$\mathrm{INH}$} & katG & 241 & 286 & 25 \\
\hline & katG promoter & 3 & 3 & 0 \\
\hline & $\operatorname{inh} A$ & 12 & 15 & 0 \\
\hline & inhA promoter & 9 & 11 & 0 \\
\hline & $a h p C$ & 8 & 8 & 0 \\
\hline & $a h p C$ promoter & 13 & 14 & 0 \\
\hline & kasA & 8 & 11 & 0 \\
\hline \multirow[t]{2}{*}{ RMP } & rpoB & 89 & 135 & 19 \\
\hline & rpoC & 8 & 8 & 0 \\
\hline \multirow[t]{5}{*}{ EMB } & $e m b B$ & 123 & 153 & 1 \\
\hline & $e m b A$ & 5 & 5 & 0 \\
\hline & embA promoter & 3 & 3 & 0 \\
\hline & $e m b C$ & 25 & 26 & 0 \\
\hline & $e m b R$ & 22 & 24 & 0 \\
\hline \multirow[t]{2}{*}{ STR } & rrs & 21 & 25 & 0 \\
\hline & rpsL & 14 & 19 & 0 \\
\hline \multirow[t]{4}{*}{ PZA } & pncA & 215 & 269 & 64 \\
\hline & pncA promoter & 4 & 6 & 0 \\
\hline & $\operatorname{rps} A$ & 3 & 4 & 0 \\
\hline & pand & 9 & 11 & 1 \\
\hline \multirow[t]{4}{*}{ ETH } & ethA & 33 & 29 & 5 \\
\hline & ethR & 3 & 4 & 0 \\
\hline & inhA promoter & 3 & 3 & 0 \\
\hline & $\operatorname{inh} A$ & 3 & 3 & 0 \\
\hline \multirow[t]{2}{*}{ FLQs } & gyrA & 15 & 22 & 0 \\
\hline & gyrB & 22 & 29 & 0 \\
\hline AMK & rrs & 8 & 9 & 0 \\
\hline \multirow[t]{2}{*}{ CAP } & rrs & 3 & 4 & 0 \\
\hline & tlyA & 26 & 18 & 10 \\
\hline \multirow[t]{2}{*}{ KAN } & rrs & 3 & 4 & 0 \\
\hline & eis promoter & 9 & 10 & 0 \\
\hline \multirow[t]{3}{*}{ PAS } & thyA & 23 & 17 & 5 \\
\hline & folc & 16 & 19 & 0 \\
\hline & $r i b B$ & 1 & 1 & 0 \\
\hline \multirow[t]{2}{*}{ LZD } & $r r l$ & 2 & 2 & 0 \\
\hline & rp/C & 1 & 1 & 0 \\
\hline BDQ CFZ & Rv0678 & 7 & 5 & 2 \\
\hline
\end{tabular}

AMK, amikacin; BDQ, bedaquiline; CAP, capreomycin; CFZ, clofazimine; EMB, ethabutol; ETH, ethionamide; FLQs, fluoroquinolones; INH, isoniazid; KAN, kanamycin; LZD, linezolid; PAS, para-aminosalycylic acid; PZ, pyrazinamide; RMP, rifampicin; STR, streptomycin.

(SNPs and indels) at 992 nucleotide positions from 31 loci, six promoters and 25 coding regions (see [22] for full list). In addition to examining individual drugs we considered the cumulative loci for MDR- and XDR-TB. 
Circos software [23] was used to construct circular genomic region variation maps. Polymorphisms associated with MDR- and XDR-TB are shown in Figure 1 (See Additional file 1: Figure S1 for full details).

\section{Sequence data and drug susceptibility testing}

The precision of the curated library for predicting resistance was assessed through analysis of new and published sequence data. In silico inferred resistance phenotypes were compared to phenotypes derived from conventional culture-based methods with the exception of PAS, LZD, CFZ and BDQ, for which insufficient phenotypic DST were available for comparison. Six geographically distinct datasets were used: China $(\mathrm{n}=161)$ [24], Karachi, Pakistan $(\mathrm{n}=42)$ [25], Karonga District, Malawi $(\mathrm{n}=337)$ [26], Lisbon and Porto, Portugal $(\mathrm{n}=208)$ [27], Samara, Russia [28] $(\mathrm{n}=21)$ and Vancouver, Canada $(\mathrm{n}=19)$ [29] (See Additional file 1: Table S3). Strains used in the study are a convenience sample and do not necessarily reflect the population at the site of collection. All collections had Illumina raw sequencing data (minimum read length $50 \mathrm{bp}$ ) and drug susceptibility data from recognised testing protocols [30]. Where conventional susceptibility data was not available, samples were excluded from analysis for that drug. Sensitivity, specificity and diagnostic accuracy (area under the receiver operating characteristic curve) were estimated using the phenotypic drug susceptibility test result as the reference standard [31]. $P$ values and confidence intervals were determined using binomial distribution approximations.

\section{Rapid mutation detection and the TB Profiler Online tool}

To rapidly characterise mutations from whole genome sequence files (fastq format), we map raw sequences to a modified version of the $\mathrm{H} 37 \mathrm{Rv}$ reference genome (Genbank accession number: NC_000962.3) using the Snap algorithm [32], and call SNPs and indels using samtool/ $v c f$ tools of high quality (Q30, 1 error per 1,000 bp) as previously described $[21,33]$. The modified reference genome consists of the genes and flanking regional sequences containing the 1,325 drug resistance mutations in the curated list (Table 1) and selected lineage specific mutations [21]. All high quality SNPs and indels identified from the alignments are compared to the curated list to determine known and novel polymorphism. Algorithmic results obtained were compared to standard SNP calling procedures using the full reference genome [21]. The online TB Profiler tool [34] was developed in Perl/PHP. It inputs raw sequence data (fastq format), identifies drug resistance and lineage specific mutations, and displays related outputs (see screenshots in

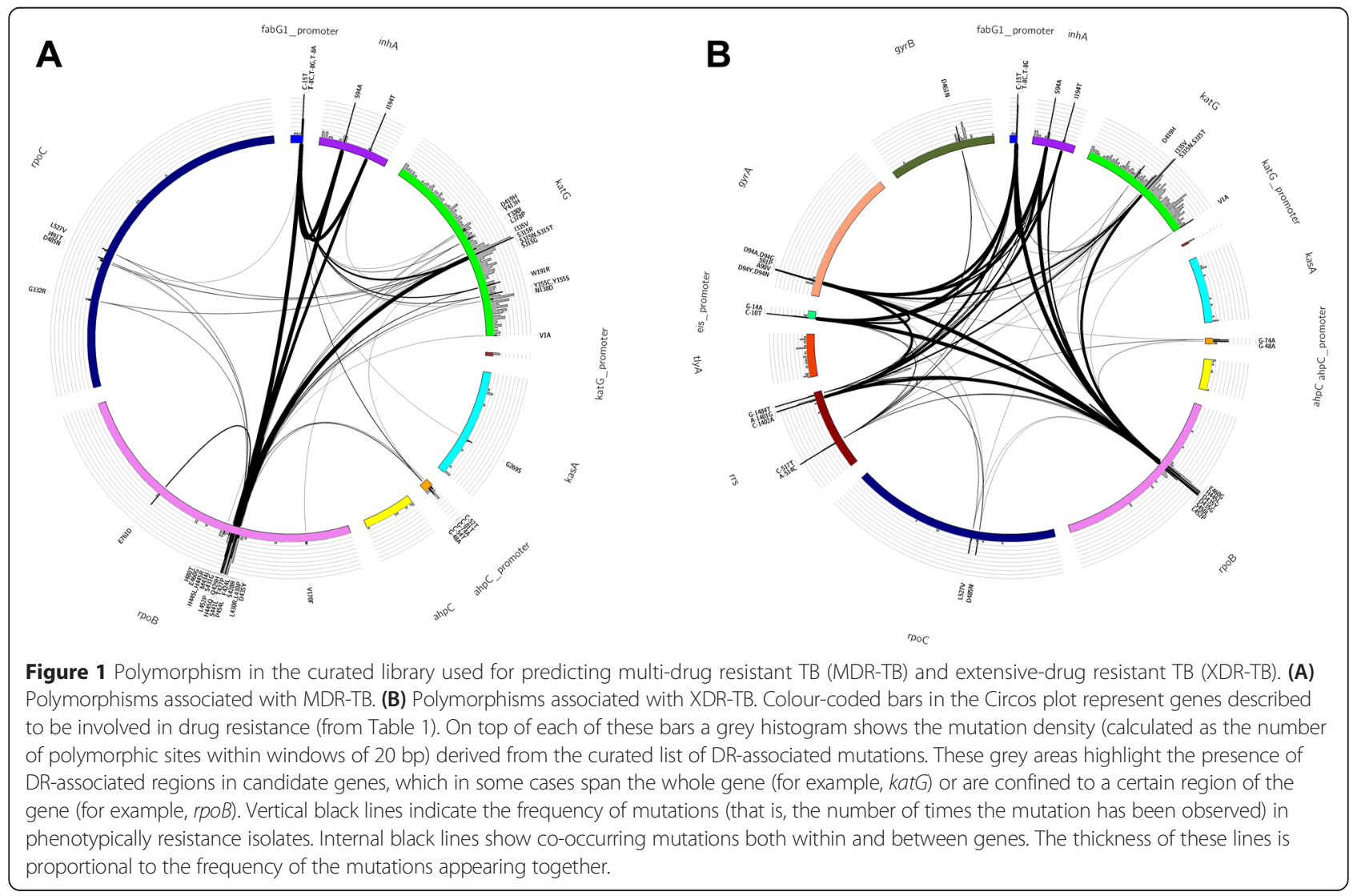


Additional file 1: Figure S2). A Perl script was used to implement the Snap software and samtool/vcf based bioinformatic pipeline. The script is available from the corresponding author.

\section{Comparison with existing tools}

To examine the potential analytical advantage of whole genome sequencing comparison was made with three commercial tests: (1) the Xpert MTB/RIF (Cepheid Inc., USA) which targets the rpoB gene for RMP resistance; (2) the LPA MTBDRplus for MDR-TB (Hain Lifescience, Germany) which targets rpo $\mathrm{B}, k a t \mathrm{G}$ and inh $\mathrm{A}$ for resistance to RMP and INH; and (3) the LPA MTBDRsl (Hain Lifescience, Germany) which targets gyrA, rrs and $e m b \mathrm{~B}$ for resistance to the fluoroquinolones (FLQ), aminoglycosides and ethambutol, respectively. In silico versions were developed based on the polymorphisms used by these assays and their performance compared to the whole genome mutation library. In particular, in silico analysis of the six datasets was performed and analytical sensitivities and specificities of the inferred resistance relative to the reported phenotype were compared (Figure 2, Additional file 1: Figures S3 and S4). KvarQ [35], a new tool that directly scans fastq files of bacterial genome sequences for known genetic polymorphisms, was run across all 792 samples using the MTBC test suite and default parameters. Sensitivity and specificity achieved by this method using phenotypic DST results as the reference standard were calculated.

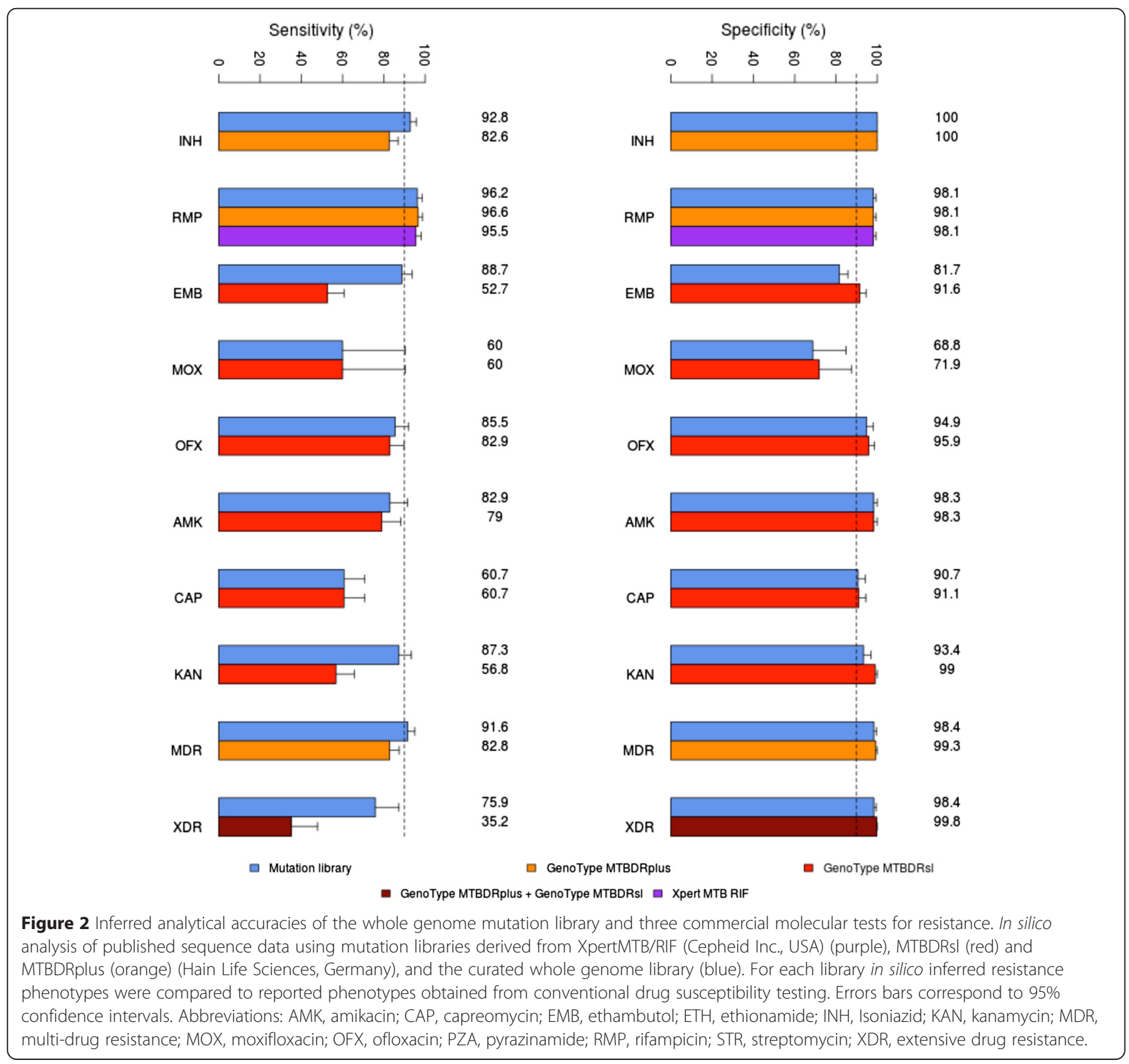




\section{Results}

\section{A mutation library}

Following review of available data (See Additional file 1: Table S1 for a list of source materials), a library comprising 1,325 polymorphisms (single nucleotide polymorphisms (SNPs) and indels) at 992 nucleotide positions from 31 loci, six promoters and 25 coding regions was established. This library covered the anti-TB drugs: EMB, ETH, INH, PZA, RMP, STR and the second line drugs used to treat MDR-TB AMK, CAP, KAN, MOX and OFX. Mutations associated with resistance to PAS, LZD, CFZ and BDQ were also compiled but were not included in the analysis given lack of available phenotypic DST results. In addition to examining individual drugs we considered the cumulative loci for MDR- and XDR-TB. Polymorphisms associated with MDR- and XDR-TB are shown in Figure 1 (see Additional file 1: Figure S1 for full details).

\section{Validation of the mutation library}

The mutation library was validated using new and publically available sequence and phenotypic data from 792 isolates, from six countries (Canada, China, Malawi, Pakistan, Portugal and Russia; see Additional file 1: Table S3). Of the 792 isolates, 365 (46\%) were phenotypically resistant to at least one drug, 262 (33\%) were MDR-TB, 54 (6.8\%) XDR-TB and 426 (54\%) were susceptible to all drugs tested. In silico genotyping [36] revealed the major modern $M$. tuberculosis lineages were represented, including Lineage 1 (East African Indian spoligotype family: 68, 8.6\%), Lineage 2 (Beijing spoligotype: 182, 23\%), Lineage 3 (Central Asian: 86, 10.9\%) and Lineage 4 (456 isolates, 57.5\% including $298 \mathrm{LAM}, 35 \mathrm{X}, 97 \mathrm{~T}, 4 \mathrm{~S}, 18 \mathrm{H}$ and 4 other spoligotypes). In silico inferred resistance from whole genome sequence data was compared to the reported resistance phenotype from conventional culture-based susceptibility testing. Results are summarised in Table 2. Sensitivity and specificity varied by drug, and with the geographic origin (Additional file 1: Figure S4). For the drugs that contribute to MDR-TB correlation of mutation analysis with the reported phenotype was high. Mutations predictive of resistance were found in $96.0 \%$ and $92.8 \%$ of samples resistant to RMP and INH, respectively. Of 22 phenotypically INH resistant samples not detected by mutation analysis, 14 were from China. Further analysis revealed seven had mutations in known candidate loci ( $k a t G$ and $a h p C$ promoter) not previously reported (Additional file 1 : Table S4). No additional cases of INH resistance were suggested by the genome analysis. However, 10 isolates reported as susceptible to RMP by conventional testing had mutations predictive of resistance, six of which were from Malawi. Correlation was slightly poorer for other first line drugs. For PZA 32 of 110 samples with a resistant phenotype were not recognised by genome analysis, including 18 of 37 samples from Karachi. However, specificity for this drug was high (93\%; 95\% CI: 90.6 to 97.2). Correlation was also reduced for EMB where 61 of 334 susceptible stains were found to harbour mutations included in the library of resistance polymorphisms $(81.7 \%$ specificity). For the aminoglycosides used to treat MDR-TB correlation was higher for AMK and KAN than for CAP, where 35 of 89 resistant samples were not detected by the in silico genome analysis. Testing for fluoroquinolone resistance was less commonly reported and data for OFX was restricted to 313 samples from two studies (China and Portugal). Mutations were not identified in 17 resistant samples (85.5\% sensitivity) and 10 drug susceptible samples were found to harbour mutations associated with resistance (94.9\% specificity). Of 42 samples tested for susceptibility to MOX, 10 were reported as phenotypically resistant, of which six were recognised by the in silico mutation analysis.

\section{Comparison with commercial tests and other drug resistance databases}

Having assessed the diagnostic potential of the mutation library, comparison was made with the polymorphisms used in commercially available molecular tests for drug resistance. Results are summarised in Figure 2. There was no significant difference between the mutation library and polymorphisms employed by the Xpert MTB/ RIF and the LPA MTBDRplus for detecting resistance to RMP. However, 31 samples had mutations predictive of resistance to INH not covered by the MTBDRplus. The alleles concerned were mainly in the gene encoding catalase-peroxidase enzyme (katG) (S315N ( $=9)$, S315G ( $\mathrm{n}=1)$, D419H $(\mathrm{n}=1), \operatorname{L378P}(\mathrm{n}=1)$, V1A $(\mathrm{n}=1)$, Y155C ( $\mathrm{n}=3)$, W191R ( $=5$ and always with C-15T inhA promoter), N138D ( $\mathrm{n}=1$, with $\mathrm{T}-8 \mathrm{~A}$ inh $A$ promoter) and T380I ( $\mathrm{n}=1$; with $\mathrm{C}$-15T inhA promoter). There were also six samples with $\operatorname{ahpC}$ promoter mutations and two samples with inhA mutations (S94A and I194T). No resistance mutations were observed in INH susceptible strains suggesting $100 \%$ specificity. Overall, when screening for MDR-TB the mutation library offered enhanced accuracy over the line probe mutations ( 95.8 vs. $93.1 \%$; $P<0.0004$ ) (Table 2).

Fewer susceptibility data were available for the second line drugs. For each of the fluoroquinolones and aminoglycosides the sensitivity of the mutation library was equal to, or greater than for the mutations employed in the LPA MTBDRsl (Figure 2), although a slight reduction in specificity was observed: MOX (71.9 vs. $68.8 \%$, $P<0.32$ ), OFX (95.9 vs. $94.9 \%, P<0.083)$, CAP (91.1 vs. $90.7 \%, P<0.32)$, KAN (99.0 vs. $93.4 \%, P<0.001)$ and EMB (86.6 vs. $81.7 \%, P<0.001)$. Overall when detecting 
Table 2 Accuracy of whole genome drug resistance analysis compared to reported resistance phenotype when applied to in silico determination of resistance from raw sequence data

\begin{tabular}{|c|c|c|c|c|c|c|c|c|c|c|c|}
\hline Drug & Sample size & Resistant (\%) & Sensitivity $(95 \% \mathrm{Cl})$ & Specificity $(95 \% \mathrm{Cl})$ & Accuracy $(95 \% \mathrm{Cl})$ & $\begin{array}{l}\text { China } \\
\text { Sen/Spec }\end{array}$ & $\begin{array}{l}\text { Pakistan } \\
\text { Sen/Spec }\end{array}$ & $\begin{array}{l}\text { Malawi } \\
\text { Sen/Spec }\end{array}$ & $\begin{array}{l}\text { Portugal } \\
\text { Sen/Spec }\end{array}$ & $\begin{array}{l}\text { Russia } \\
\text { Sen/Spec }\end{array}$ & $\begin{array}{l}\text { Canada } \\
\text { Sen/Spec }\end{array}$ \\
\hline $\mathrm{INH}$ & 693 & $305(44)$ & $92.8(89.9-95.7)$ & $100(100-100)$ & $96.8(95.5-98.1)$ & $88.0 / 100$ & $100 / 100$ & $92.6 / 100$ & $94.6 / 100$ & $100 / 100$ & $-/ 100$ \\
\hline RMP & 694 & $264(38)$ & $96.2(93.9-98.5)$ & $98.1(96.8-99.4)$ & $97.4(96.2-98.5)$ & $95.7 / 97.7$ & $97.3 / 100$ & $100 / 98.2$ & $96.9 / 100$ & $90.9 / 90.0$ & $-/ 100$ \\
\hline EMB & 484 & $150(31)$ & $88.7(83.6-93.8)$ & $81.7(77.6-85.8)$ & $83.9(80.6-87.2)$ & $83.6 / 71.3$ & $100 / 42.7$ & $100 / 80$ & $85.7 / 68.1$ & $100 / 80.0$ & $-/ 100$ \\
\hline STR & 487 & $225(46.2)$ & $87.1(82.7-91.5)$ & $89.7(86.0-93.4)$ & 88.5 (85.7-91.3) & $86.8 / 91.0$ & $95.8 / 44.4$ & $61.5 / 95.6$ & $86.8 / 81.5$ & $100 / 100$ & $-/ 100$ \\
\hline PZA & 307 & $110(35.8)$ & $70.9(62.4-79.4)$ & $93.9(90.6-97.2)$ & $85.7(81.7-89.6)$ & NT & $51.3 /-$ & $66.7 / 94.8$ & $80.6 / 100$ & $100 / 60.0$ & $-/ 100$ \\
\hline ETH & 334 & $155(46.4)$ & $73.6(66.7-80.5)$ & $93.3(89.6-97.0)$ & $84.1(80.2-88.1)$ & $38.9 / 97.3$ & $66.7 / 90.3$ & NT & $84.9 / 84.6$ & NT & NT \\
\hline MOX & 42 & $10(23.8)$ & $60.0(29.6-90.4)$ & $68.7(52.6-84.8)$ & $66.7(52.4-80.9)$ & NT & NT & NT & $83.3 / 56.2$ & $25.0 / 100$ & NT \\
\hline OFX & 313 & $117(37.4)$ & 85.5 (79.1-91.9) & $94.9(91.8 \cdot 98.0)$ & $91.4(88.3-94.5)$ & $77.8 / 95.1$ & $-/ 100$ & NT & $92.1 / 93.2$ & NT & NT \\
\hline AMK & 193 & $76(39.4)$ & $82.9(74.4-91.4)$ & $98.3(96.0-100)$ & $92.2(88.4-96.0)$ & NT & $86.5 / 100$ & NT & $79.5 / 98.2$ & NT & NT \\
\hline CAP & 358 & $89(24.9)$ & $60.7(50.6-70.8)$ & $90.7(87.2-94.2)$ & $83.2(79.4-87.1)$ & $50.0 / 97.0$ & $85.7 / 21.7$ & NT & $57.7 / 98.0$ & $100 / 91.7$ & NT \\
\hline KAN & 118 & $118(37.3)$ & $87.3(81.3-93.3)$ & $93.4(89.9-96.9)$ & 91.1 (88.0-94.3) & 71.4/97.0 & 83.8/- & NT & $98.0 / 88.7$ & $80.0 / 33.3$ & NT \\
\hline MDR & 693 & $262(37.8)$ & $91.2(87.8-94.6)$ & $98.4(97.2-99.6)$ & $95.8(94.3-97.3)$ & $86.3 / 100$ & $97.3 / 100$ & $100 / 98.2$ & $95.8 / 100$ & $90.9 / 90.0$ & $-/ 100$ \\
\hline XDR & 601 & $54(9)$ & $75.9(64.5-87.3)$ & 98.4 (97.3-99.5) & 96.3 (94.8-97.8) & $60.9 / 99.1$ & $-/ 100$ & $-/ 100$ & $96.3 / 88.9$ & 25.0/100 & $-/ 100$ \\
\hline
\end{tabular}

AMK, amikacin; CAP, capreomycin; Cl, confidence interval; EMB, ethambutol; ETH, ethionamide; INH, isoniazid; KAN, kanamycin; MDR, multi-drug resistance; MOX, moxifloxacin; OFX, ofloxacin; NT, not tested;

PZA, pyrazinamide; RMP, rifampicin; Sen, sensitivity; Spec, specificity; STR, streptomycin; XDR, extensive drug resistance. 
XDR-TB the whole genome analysis offered enhanced accuracy over the line probe assay (96.3 vs. $93.7 \% ; P<0.0047$ ) (Table 2).

The mutation library was also found to be more accurate than previously reported databases $T B D r e a M D B$ and $M U B I I-T B-D B$ (Additional file 1: Figure S3), because of false positive resistance arising in those databases due to the inclusion of some phylogenetic (but not drug resistance) informative SNPs. An improvement in sensitivity was also achieved for INH, EMB, ETH, PZA and KAN by considering recently discovered drug resistance loci and polymorphisms (Additional file 1: Figure S3).

When compared to KvarQ [35] the mutation library achieved higher sensitivity for resistance to isoniazid, pyrazinamide, ofloxacin and amikacin with increases of $5.9 \%$, $8.2 \%, 3.5 \%$ and $7.9 \%$, respectively, without compromising specificity (Additional file 1: Table S5). Higher sensitivity was also achieved for ethambutol (28\%), streptomycin (7.1\%) and kanamycin (33.1\%) but with reductions in specificity $(-7.5 \%,-9.1 \%$ and $-5.1 \%$, respectively). Sensitivity and specificity values remained the same or very similar for rifampicin and moxifloxacin. KvarQ did not predict resistance status for ethionamide and capreomycin.

\section{Online tool for predicting drug resistance and lineage information from sequenced isolates}

Having established a curated list of 1,325 mutations predictive of resistance, we sought to develop a web-based tool to rapidly identify a DST and strain-type profile. Our approach called 'TB Profiler' ([34], Additional file 1: Figure S2) aligns raw sequencing data to an abridged reference genome covering genomic regions of interest. The alignment is robust to indels and genomic frameshifts, and can be completed in minutes. Detection of $M$. tuberculosis lineage specific markers was also incorporated [21]. In addition to identifying known drug resistance associated mutations, the tool also identifies other mutations in the candidate regions. TB profiler processed fastq files at a linear rate of 80,000 sequence reads per second. Application to the 792 samples led to the identification of 38 novel mutations (24 nonsynonymous SNPs, 9 indels and 5 intergenic SNPs) present in phenotypically resistant strains but absent in susceptible ones (Additional file 1: Table S4). All mutations were confirmed by the alignment of the short reads to the whole H37Rv genome reference sequence using established genome analysis pipelines [21]. The median run-time for the TB Profiler was $5 \mathrm{~min}$ (range, 2 to $10 \mathrm{~min}$ ) across samples with depth of coverage ranging from 20 - to 1,000-fold. TB Profiler can also be downloaded and run locally in a Unix environment [37].

\section{Discussion}

The emergence and amplification of resistance to antituberculosis drugs has created a need for improved detection tools to guide treatment options for patients with MDR-TB, XDR-TB and post XDR (TDR-TB) disease. Molecular-based drug-susceptibility tests are more rapid and microbiologically safe compared to phenotypic assays. Nonetheless, rapid molecular assays are currently limited. GeneXpert (Cepheid) tests only for rifampicin resistance, the sensitivity of GenoType MTBDRplus (Hain Life-Science) for the detection of isoniazid resistance is reported to be approximately $80 \%$ to $90 \%[38,39]$ and the GenoType MTBDRsl assay performs inadequately for fluoroquinolones, aminoglycosides and ethambutol (reported sensitivities of $87 \%$ to $89 \%, 21 \%$ to $100 \%$ and $39 \%$ to $57 \%$, respectively) [40,41]. Wholegenome sequencing has the potential to determine the full antibiogram if the genetic determinants of antibiotic resistance are known [15-17,42]. However, M. tuberculosis sequencing has mainly been performed from cultures and sequencing directly from clinical specimens such as sputum still needs to be optimised. Compared to Sanger sequencing that requires multiple sequencing reactions to cover the various drug resistance loci, whole-genome sequencing has the ability to characterise all nucleotide positions in a single experiment. The depth of next generation sequencing, where each loci is examined numerous times (typically 100-fold coverage) provides capacity to detect genetically mixed bacterial populations (hetero-resistance) [43].

We have compiled and released a mutation library for M. tuberculosis drug resistance [22]. By comparing in silico drug resistance predictions to conventional phenotypic results, we have demonstrated that our library is more accurate than current commercial molecular tests and alternative mutation databases. Combining the mutation library with a rapid detection tool for whole sequencing data [34], we have demonstrated the potential for using next generation sequencing for detecting drug resistance.

In silico validation of the mutation library demonstrated high sensitivity for detecting resistance to RMP, with the majority of resistance mutations found in a single region of the rpoB gene [44]. Unsurprisingly, the mutation analysis was less reliable for drugs with more complex modes of action and where knowledge of the genetic basis of resistance is less complete (for example, PZA, ETH and EMB). Still, our curated library was more accurate during in silico analysis for MDR and XDR-TB than the commercial line probe assays, in addition to assessing a greater number of drugs. Improved sensitivity was reported for INH, AMK, EMB, PZA and KAN (Figure 2 and Additional file 1: Figure S3). The inferred diagnostic performance from whole genome sequences for the commercial tests may be overestimated, as in a real scenario these tests have low detection limits and are unable to differentiate synonymous from nonsynonymous amino acid changes [11]. 
A limiting factor for this study is the reliability of culture-based susceptibility testing methods, particularly those for EMB and PZA, and the lack of a consensus reference standard with which to compare new tests. Future studies should be encouraged to adopt standardised quantitative phenotypic assays [45]. DST is particularly problematic for PZA [46] and false resistance results are not uncommon [46]. The pncA gene (involved in resistance to PZA) is one of the most polymorphic genes in the $M$. tuberculosis genome and attempts to increase sensitivity by including additional SNPs resulted in a reduction in specificity. Further work is needed to determine additional resistance polymorphisms, including validation of putative markers with high quality phenotypic and clinical data. It should be noted that high positive predictive values are crucial for drug resistance tests where the consequence of a false positive may be unnecessary treatment with drugs of high toxicity and prolonged isolation in dedicated containment facilities. Although an important increase in sensitivity was achieved for EMB (88.7\%), the specificity of $81.7 \%$ is poor. These results concur with suggestions that degrees of resistance to EMB may be acquired through mutations in multiple loci, some of which are currently unknown [47]. Although current knowledge does not allow EMB resistance to be predicted with high precision, known mutations may be used to identify strains predisposed to developing high-level resistance. Our results demonstrate the considerable cross-resistance between the fluoroquinolones. Minimal inhibitory concentrations (MIC) can vary for these drugs and information on specific polymorphisms may influence dosing levels [48].

The poor specificity obtained for CAP and EMB may be explained in terms of the high MIC used to classify strains as clinically resistant or susceptible. Strains having MIC values slightly below this cutoff have genetically detectable resistance mechanisms but will falsely be identified as susceptible $[45,49]$. Low specificity was also obtained for MOX (68.7\%) as opposed to that of OFX (94.9\%) using the same fluoroquinolones resistance markers (that is, $g y r A$ and $g y r B$ mutations). Mutations in gyrA and $g y r B$ confer resistance to fluoroquinolones, albeit not at the same level, with MOX normally presenting the lowest MIC values in the group followed by levofloxacin, in contract with the higher levels of resistance observed for OFX and ciprofloxacin [50]. Strains having the same fluoroquinolones resistance-conferring mutations are therefore more likely to be regarded as sensitive phenotypically (false positives) for MOX leading to lower specificity values. However, caution should be exercised when considering the MOX data as few phenotypic results were available and the uncertainty of analysis is reflected in the wide confidence intervals reported.
The accuracy of the mutation analysis was observed to vary by geographic region (Additional file 1: Figure S4). Geographic disparities in the frequency of drug resistant SNPs may reflect local treatment strategies and the clonal nature of tuberculosis transmission and therefore be the result of local microevolution. It has previously been suggested that emergence of resistance in M. tuberculosis is associated with bacterial lineage. However, such conclusions cannot be drawn from the present study, as sampling strategies were not appropriate to such analysis.

Not all drugs used in the treatment of tuberculosis were included in this study. Drugs were omitted either because insufficient susceptibility data were available (that is, PAS, LZD, CFZ and BDQ) or because the mechanism of action remains obscure and SNPs to predict resistance have yet to be systematically identified (for example, cycloserine). A major advantage of the whole genome approach is that all data are captured and additional loci can easily be incorporated in the mutation library. Future work should assess the diagnostic accuracy of drug resistance mutations identified for PAS, LZD, CFZ and BDQ in clinical specimens.

Previous studies on discrepancies between mutation and culture-derived resistant phenotypes suggest that molecular assessment may eventually become the reference standard for some drugs [51,52]. We have demonstrated rapid analysis of whole genome sequence data to provide the genotype and predict resistance to 11 antiTB drugs. In the absence of whole genome sequencing technology, which is still prohibitive in low-resource settings, drug resistance markers can be detected using alternative genotyping platforms, such as multiplex ligation-dependent probe amplification (MLPA) assays [53] or multiplexed oligonucleotides ligation PCR [54]. The presented curated database will facilitate the development of more accurate molecular drug-susceptibility tests.

Rapid determination of strain-specific and drug resistance mutations will be beneficial for therapeutic selection, clinical management of patients and implementation of infection control measures. The free-to-use TB Profiler prototype is available for a research setting, and further studies are needed to assess its performance for clinical use.

\section{Conclusion}

We have constructed an on-line software tool and methodology that provides rapid analysis of genome sequence data to describe the lineage of the $M$. tuberculosis strain under test and predict resistance to 11 anti-TB drugs. The tool refers to a library comprising 1,325 mutations that is the most comprehensive and accurate such data source yet reported. In addition to providing information 
about a greater number of drugs, a whole genome approach has the potential to improve detection sensitivity for drugs such as isoniazid over the currently available molecular tests. The ability to analyse raw sequence data and extract information of clinical relevance in a few minutes would render whole genome analysis faster than current phenotypic testing methods. Accelerated access to tailored treatment could improve cure rates and reduce exposure to ineffective toxic drugs, improving the patient experience and facilitating compliance. The analytical methodology described is flexible to allow moderation of the library to encompass novel mutations and incorporate new drugs should the need arise.

\section{Additional file}

Additional file 1: Table S1. Sources of $M$. tuberculosis drug resistance polymorphisms used to curate a mutation database. Table S2. Phylogenetic SNPs not included in the curated database. Table S3. Summary of Mycobacterium tuberculosis whole genome sequence datasets used in this study. Table S4. Novel mutations identified by TB profiler. Table S5. Diagnostic performance of TB profiler compared to KvarQ method. Figure S1. Circos plots summarising drug resistant associated genes and mutations in the curated library for anti-tuberculosis drugs. Figure S2. The TB profiler tool (http://tbdr.lshtm.ac.uk) - Screenshot of TB profiler input page. Figure S3. Diagnostic performance of the curated library compared to alternative drug resistance mutation databases, using phenotype drug susceptibility data as the reference standard. Figure S4. Diagnostic accuracy across populations.

\section{Abbreviations}

AMK: amikacin; BDQ: bedaquiline; CAP: capreomycin; CFZ: clofazimine; DR: Drug resistance; DST: Drug Susceptibility testing; EMB: ethambutol; ETH: ethionamide; INH: isoniazid; KAN: kanamycin; LPA: Line Probe Assays; LZD: linezolid; MDR-TB: Multi drug-resistant Tuberculosis; MIC: Minimal inhibitory concentrations; MLPA: ligation-dependent probe amplification; MOX: moxifloxacin; indels: insertion and deletions; OFX: ofloxacin; PAS: para-aminosalicylic acid; PZA: pyrazinamide; RMP: rifampicin; SNP: single nucleotide polymorphism; STR: streptomycin; TB: tuberculosis; TDR-TB: totally drug resistant tuberculosis, XDR-TB, extensively drug resistant tuberculosis; WHO: World Health Organization

\section{Competing interests}

The authors declare that they have no competing interests.

\section{Authors' contributions}

FC, RM, AP and TGC conceived and designed the study; FC and MDP developed and tested the online tool; JAG-A and AW developed additional software; GH-C, MN and KM performed laboratory experiments and curation of meta data for sequencing; $A \mathrm{~A}, \mathrm{AM}, \mathrm{JP}, \mathrm{MV} I \mathrm{P} Z \mathrm{ZH}, \mathrm{RH}$ and JRG contributed biological samples, sequencing or phenotypic data; FC performed the statistical analysis under the guidance of NM and TGC; AP led the sequencing efforts; FC, RM and TGC wrote/drafted and finalised the manuscript with contributions from all other authors. The final manuscript was read and approved by all authors.

\section{Acknowledgements}

This work was funded by the Bloomsbury Research Fund, Fundação para a Ciência e Tecnologia, King Abdullah University of Science and Technology (KAUST), Medical Research Council UK (grant number MR/K000551/1) and the Wellcome Trust (grant number 096249/Z/11/B).

\section{Author details}

'Faculty of Infectious and Tropical Diseases, London School of Hygiene \& Tropical Medicine, Keppel Street, London WC1E 7HT, UK. ${ }^{2}$ Advanced Data
Analysis Centre, University of Nottingham, Wollaton Road, Nottingham NG8 1BB, UK. ${ }^{3}$ Biological and Environmental Sciences and Engineering Division, King Abdullah University of Science and Technology (KAUST), Thuwal, Kingdom of Saudi Arabia. ${ }^{4}$ Sydney Emerging Infections and Biosecurity Institute and School of Public Health, University of Sydney, Sydney, Australia. ${ }^{5}$ Tuberculosis Laboratory, Instituto Nacional de Saude Dr. Ricardo Jorge, Porto, Portugal. ${ }^{6}$ Centro de Patogénese Molecular, Faculdade de Farmácia da Universidade de Lisboa, Lisbon, Portugal. ${ }^{7}$ Grupo de Micobactérias, Unidade de Microbiologia Médica, Instituto de Higiene e Medicina Tropical, Universidade Nova de Lisboa, Lisbon, Portugal. ${ }^{8}$ Department of Pathology \& Microbiology, Aga Khan University Hospital, Karachi, Pakistan. ${ }^{9}$ Karonga Prevention Study, Chilumba, Malawi. ${ }^{10}$ Department of Computer Science, Birkbeck College, University of London, Malet Street, London WC1E 7HX, UK.

Received: 23 December 2014 Accepted: 16 April 2015

Published online: 27 May 2015

\section{References}

1. Dheda K, Gumbo T, Gandhi NR, Murray M, Theron G, Udwadia Z, et al. Global control of tuberculosis: from extensively drug-resistant to untreatable tuberculosis. Lancet Respir Med. 2014;2:321-38.

2. Bastos ML, Hussain H, Weyer K, Garcia-Garcia L, Leimane V, Leung CC, et al. Treatment outcomes of patients with multidrug-resistant and extensively drug-resistant tuberculosis according to drug susceptibility testing to firstand second-line drugs: an individual patient data meta-analysis. Clin Infect Dis. 2014;59:1364-74.

3. Pooran A, Pieterson E, Davids M, Theron G, Dheda K. What is the cost of diagnosis and management of drug resistant tuberculosis in South Africa? PLoS One. 2013:8:e54587.

4. Yee D, Valiquette C, Pelletier M, Parisien I, Rocher I, Menzies D. Incidence of serious side effects from first-line antituberculosis drugs among patients treated for active tuberculosis. Am J Respir Crit Care Med. 2003;167:1472-7.

5. Lange C, Abubakar I, Alffenaar J-WC, Bothamley G, Caminero JA, Carvalho ACC, et al. Management of patients with multidrug-resistant/extensively drug-resistant tuberculosis in Europe: a TBNET consensus statement. Eur Respir J. 2014;44:23-63.

6. Shean K, Streicher E, Pieterson E, Symons G, van ZyI SR, Theron G, et al. Drug-associated adverse events and their relationship with outcomes in patients receiving treatment for extensively drug-resistant tuberculosis in South Africa. PLoS One. 2013;8:e63057.

7. Zhang Y, Yew WW. Mechanisms of drug resistance in Mycobacterium tuberculosis. Int J Tuberc Lung Dis. 2009;13:1320-30.

8. Food US, Administration D. Xpert MTB/RIF assay 510(k) decision summary. Silver Spring, MD: US FDA; 2013 [http://www.accessdata.fda.gov/cdrh_docs/ reviews/k131706.pdf]

9. Ling DI, Zwerling AA, Pai M. GenoType MTBDR assays for the diagnosis of multidrug-resistant tuberculosis: a meta-analysis. Eur Respir J. 2008:32:1165-74.

10. Ajbani K, Nikam C, Kazi M, Gray C, Boehme C, Balan K, et al. Evaluation of genotype MTBDRsl assay to detect drug resistance associated with fluoroquinolones, aminoglycosides and ethambutol on clinical sediments. PLoS One. 2012;7:e49433.

11. Alonso M, Palacios JJ, Herranz M, Penedo A, Menéndez A, Bouza E, et al. Isolation of Mycobacterium tuberculosis strains with a silent mutation in rpoB leading to potential misassignment of resistance category. J Clin Microbiol. 2011:49:2688-90.

12. Jin J, Shen Y, Fan X, Diao N, Wang F, Wang S, et al. Underestimation of the resistance of Mycobacterium tuberculosis to second-line drugs by the new GenoType MTBDRsI test. J Mol Diagn. 2013;15:44-50.

13. Aubry A, Sougakoff W, Bodzongo P, Delcroix G, Armand S, Millot G, et al. First evaluation of drug-resistant mycobacterium tuberculosis clinical isolates from Congo revealed misdetection of fluoroquinolone resistance by line probe assay Due to a double substitution T80A-A90G in GyrA. PLoS One. 2014;:9:e95083

14. Köser CU, Ellington MJ, Cartwright EJP, Gillespie SH, Brown NM, Farrington $M$, et al. Routine use of microbial whole genome sequencing in diagnostic and public health microbiology. PLoS Pathog. 2012;8:e1002824.

15. Outhred AC, Jelfs P, Suliman B, Hill-Cawthorne G, Crawford ABH, Marais BJ, et al. Added value of whole-genome sequencing for management of highly drug-resistant TB. J Antimicrob Chemother. 2014:1-5. 
16. Köser CU, Bryant JM, Becq J, Török ME, Ellington MJ, Marti-Renom MA, et al. Whole-genome sequencing for rapid susceptibility testing of $\mathrm{M}$. tuberculosis. N Engl J Med. 2013;369:290-2.

17. Witney A, Gould K, Arnold A, Coleman D, Delgado R, Dhillon J, et al. Clinical application of whole genome sequencing to inform treatment for multidrug resistant tuberculosis cases. J Clin Microbiol. 2015;53:1473-83.

18. Sandgren A, Strong M, Muthukrishnan P, Weiner BK, Church GM, Murray MB. Tuberculosis drug resistance mutation database. PLoS Med. 2009;6:e1000002

19. Flandrois J-P, Lina G, Dumitrescu O. MUBII-TB-DB: a database of mutations associated with antibiotic resistance in Mycobacterium tuberculosis. BMC Bioinformatics. 2014;15:107.

20. Feuerriegel S, Köser CU, Niemann S. Phylogenetic polymorphisms in antibiotic resistance genes of the Mycobacterium tuberculosis complex. J Antimicrob Chemother. 2014;69:1205-10.

21. Coll F, McNerney R, Guerra-Assunção JA, Glynn JR, Perdigão J, Viveiros M, et al. A robust SNP barcode for typing Mycobacterium tuberculosis complex strains. Nat Commun. 2014;5:4812.

22. The mutation library used by the TB Profiler tool. [http://pathogenseq.Ishtm.ac.uk/ rapiddrdata]

23. Krzywinski M, Schein J, Birol I, Connors J, Gascoyne R, Horsman D, et al. Circos: an information aesthetic for comparative genomics. Genome Res. 2009:19:1639-45

24. Zhang H, Li D, Zhao L, Fleming J, Lin N, Wang T, et al. Genome sequencing of 161 Mycobacterium tuberculosis isolates from China identifies genes and intergenic regions associated with drug resistance. Nat Genet. 2013:45:1255-60.

25. Ali A, Hasan Z, McNerney R, Mallard K, Hill-Cawthorne G, Coll F, et al. Whole genome sequencing based characterization of extensively drug-resistant mycobacterium tuberculosis isolates from Pakistan. PLoS One. 2015;10:e0117771

26. Guerra-Assunção J, Crampin A, Houben R, Mzembe T, Mallard K, Coll F, et al, Large-scale whole genome sequencing of $M$. tuberculosis provides insights into transmission in a high prevalence area. Elife. 2015;4:e05166.

27. Perdigão J, Silva H, Machado D, Macedo R, Maltez F, Silva C, et al. Unraveling Mycobacterium tuberculosis genomic diversity and evolution in Lisbon, Portugal, a highly drug resistant setting. BMC Genomics. 2014;15:991.

28. Casali N, Nikolayevskyy V. Microevolution of extensively drug-resistant tuberculosis in Russia. Genome Res. 2012:22:735-45.

29. Gardy JL, Johnston JC, Ho Sui SJ, Cook VJ, Shah L, Brodkin E, et al. Wholegenome sequencing and social-network analysis of a tuberculosis outbreak. N Engl J Med. 2011;364:730-9.

30. World Health Organization. Guidelines for surveillance of drug resistance in tuberculosis. Geneva: WHO; 2009. [http://whqlibdoc.who.int/publications/ 2009/9789241598675_eng.pdf]

31. Altman DG. Practical statistics for medical research. 1st ed. London: Chapman and Hall/CRC Press; 1999.

32. Zaharia M, Bolosky WJ, Curtis K, Fox A, Patterson D, Shenker S, et al. Faster and more accurate sequence alignment with SNAP. Ithaca, NY: Cornell University; 2011. [www.arxiv.org/abs/1111.5572]

33. Coll F, Preston M, Guerra-Assunção JA, Hill-Cawthorn G, Harris D, Perdigão J, et al. PolyTB: A genomic variation map for Mycobacterium tuberculosis. Tuberculosis. 2014;94:346-54.

34. TB Profiler online tool. [http://tbdr.lshtm.ac.uk]

35. Steiner A, Stucki D, Coscolla M, Borrell S, Gagneux S. KvarQ: targeted and direct variant calling from fastq reads of bacterial genomes. BMC Genomics. 2014;15:881

36. Coll F, Mallard K, Preston MD, Bentley S, Parkhill J, McNerney R, et al. SpolPred: rapid and accurate prediction of Mycobacterium tuberculosis spoligotypes from short genomic sequences. Bioinformatics. 2012;28:2991-3.

37. Downloadable version of TB Profiler. [http://pathogenseq.Ishtm.ac.uk/ rapiddrcode]

38. Cavusoglu C, Turhan A, Akinci P, Soyler I. Evaluation of the Genotype MTBDR assay for rapid detection of rifampin and isoniazid resistance in Mycobacterium tuberculosis isolates. J Clin Microbiol. 2006;44:2338-42.

39. Somoskovi A, Dormandy J, Mitsani D, Rivenburg J, Salfinger M. Use of smear-positive samples to assess the PCR-based genotype MTBDR assay for rapid, direct detection of the Mycobacterium tuberculosis complex as well as its resistance to isoniazid and rifampin. J Clin Microbiol. 2006:44:4459-63.
40. Hillemann D, Rüsch-Gerdes S, Richter E. Feasibility of the GenoType MTBDRsl assay for fluoroquinolone, amikacin-capreomycin, and ethambutol resistance testing of Mycobacterium tuberculosis strains and clinical specimens. J Clin Microbiol. 2009:47:1767-72

41. Huang W-L, Chi T-L, Wu M-H, Jou R. Performance assessment of the GenoType MTBDRsI test and DNA sequencing for detection of second-line and ethambutol drug resistance among patients infected with multidrugresistant Mycobacterium tuberculosis. J Clin Microbiol. 2011;49:2502-8.

42. Köser CU, Ellington MJ, Peacock SJ. Whole-genome sequencing to control antimicrobial resistance. Trends Genet. 2014;30:401-7.

43. Eilertson B, Maruri F, Blackman A, Herrera M, Samuels DC, Sterling TR. High proportion of heteroresistance in gyrA and gyrB in fluoroquinolone-resistant Mycobacterium tuberculosis clinical isolates. Antimicrob Agents Chemother. 2014;58:3270-5.

44. Ramaswamy S, Musser JM. Molecular genetic basis of antimicrobial agent resistance in Mycobacterium tuberculosis: 1998 update. Tuber Lung Dis. 1998;79:3-29.

45. Cambau E, Viveiros M, Machado D, Raskine L, Ritter C, Tortoli E, et al. Revisiting susceptibility testing in MDR-TB by a standardized quantitative phenotypic assessment in a European multicentre study. J Antimicrob Chemother. 2014;70:686-96.

46. Zhang $Y$, Mitchison D. The curious characteristics of pyrazinamide: a review. Int J Tuberc Lung Dis. 2003;7:6-21.

47. Safi H, Lingaraju S, Amin A, Kim S, Jones M, Holmes M, et al. Evolution of high-level ethambutol-resistant tuberculosis through interacting mutations in decaprenylphosphoryl- $\beta$-D-arabinose biosynthetic and utilization pathway genes. Nat Genet. 2013;45:1190-7.

48. Maruri F, Sterling TR, Kaiga AW, Blackman A, van der Heijden YF, Mayer C, et al. A systematic review of gyrase mutations associated with fluoroquinoloneresistant Mycobacterium tuberculosis and a proposed gyrase numbering system. J Antimicrob Chemother. 2012;67:819-31.

49. Ängeby K, Juréen P, Kahlmeter G, Hoffner SE, Schön T. Challenging a dogma: antimicrobial susceptibility testing breakpoints for Mycobacterium tuberculosis. Bull World Health Organ. 2012;90:693-8.

50. Malik S, Willby M, Sikes D, Tsodikov OV, Posey JE. New insights into fluoroquinolone resistance in Mycobacterium tuberculosis: functional genetic analysis of gyrA and gyrB mutations. PLoS One. 2012;7:e39754.

51. Rigouts L, Gumusboga M, de Rijk WB, Nduwamahoro E, Uwizeye C, de Jong $B$, et al. Rifampin resistance missed in automated liquid culture system for Mycobacterium tuberculosis isolates with specific rpoB mutations. J Clin Microbiol. 2013:51:2641-5.

52. Van Deun A, Aung KJM, Bola V, Lebeke R, Hossain MA, de Rijk WB, et al. Rifampin drug resistance tests for tuberculosis: challenging the gold standard. J Clin Microbiol. 2013;51:2633-40.

53. Bergval I, Sengstake S, Brankova N, Levterova V, Abadía E, Tadumaze N, et al. Combined species identification, genotyping, and drug resistance detection of Mycobacterium tuberculosis cultures by MLPA on a bead-based array. PLoS One. 2012;7:e43240.

54. Stucki D, Malla B, Hostettler S, Huna T, Feldmann J, Yeboah-Manu D, et al. Two new rapid SNP-typing methods for classifying Mycobacterium tuberculosis complex into the main phylogenetic lineages. PLoS One. 2012;7:e41253.

\section{Submit your next manuscript to BioMed Central and take full advantage of:}

- Convenient online submission

- Thorough peer review

- No space constraints or color figure charges

- Immediate publication on acceptance

- Inclusion in PubMed, CAS, Scopus and Google Scholar

- Research which is freely available for redistribution 